\title{
Propuesta evaluativa para el Taller de Diseño Centrado en el Usuario (DCU), en la Carrera de Diseño Industrial de la Universidad del Bío-Bío, Chile
}

\author{
Juan C. Briede ${ }^{(1)}$ y Marcela L. Mora ${ }^{(2)}$ \\ (1) Universidad del Bío-Bío, Depto. de Arte y Tecnologías del Diseño, Avda. Collao 1202, Casilla 5-C, \\ Concepción-Chile (e-mail: jbriede@ubiobio.cl). \\ (2) Universidad del Bío-Bío, Área de Desarrollo Pedagógico, Unidad de Gestión Curricular y Monitoreo, \\ Avda. Andrés Bello s/n, Casilla 447, Chillán-Chile (e-mail: mamora@ubiobio.cl).
}

Recibido Sep. 14, 2012; Aceptado Nov. 13, 2012; Versión final recibida Dic. 02, 2012

\begin{abstract}
Resumen
Se presenta una propuesta evaluativa para el Taller de Diseño Centrado en el Usuario (DCU) del tercer año del ciclo formativo de la carrera de Diseño Industrial en la Universidad del Bío-Bío en Chile. La propuesta se determinó mediante el análisis y descripción de cada fase del diseño de un producto, considerando el enfoque disciplinario DCU y la metodología de proyecto. Esto con el fin de establecer indicadores evaluativos vinculados con los resultados de aprendizaje planteados por el programa del taller y con las competencias genéricas promovidas por la universidad en el marco de su responsabilidad social. La propuesta permite contar con un instrumento de evaluación objetivo en el ámbito del diseño que considera las habilidades que el estudiante debe desarrollar en cada fase del proceso de la metodología planteada. Adicionalmente, constituye un aporte en el ámbito educativo de ésta área, para mejorar los procesos evaluativos, que hasta ahora son más bien de carácter subjetivo.
\end{abstract}

Palabras clave: indicadores de evaluación, evaluación por competencias, diseño centrado en el usuario, diseño industrial, responsabilidad social.

\section{Evaluative Proposal for the User-Centered Design Workshop (UCD) in the Industrial Design Major of the University of Bío- Bío, Chile}

\begin{abstract}
An evaluative proposal for the user-centered design workshop (UCD) which is part of the third year in the formative cycle of the Industrial Design major at the Universidad del Bío-Bío in Chile is proposed. Taking into consideration the UCD disciplinary approach and project methodology. This is done to establish evaluative indicators linked to learning outcomes set by the workshop program and generic skills linked that the university promotes within its mission of social responsibility. The proposal allows having a tool for objective assessment in the field of design, which considers the skills that students must develop in each phase of the proposed methodology. Additionally, it is a contribution to the education in this area with the aim of improving the evaluative processes, which up until now have been mainly of subjective character.
\end{abstract}

Keywords: evaluation indicators, evaluation according to skills, user-centered design, Industrial design, social responsibility 


\section{INTRODUCCIÓN}

La sociedad actual, delega en el contexto universitario la función de formar en los estudiantes las competencias necesarias que les permitan desempeñarse en los diversos campos laborales (Ospina, 2006), entendiendo por competencias (Regalado et al., 2011), la aptitud para enfrentar eficazmente una familia de situaciones análogas, movilizando a conciencia y de manera a la vez rápida, pertinente y creativa, múltiples recursos cognitivos: saberes, capacidades, micro-competencias, informaciones, valores, actitudes, esquemas de percepción, de evaluación y de razonamiento, (Perrenoud, 2004). En este ámbito, el formar en competencias involucra hacer cambios significativos en los procesos evaluativos (Cano, 2008), integrando las competencias disciplinarias del Diseñador Industrial, como las genéricas, que en este caso será la Responsabilidad Social, por vincularse con un contexto de vulnerabilidad social. Por ello la evaluación es catalogada como reguladora del aprendizaje, puesto que la retroalimentación durante el proceso, la posibilidad de mejora, la autoevaluación y la evaluación de compañeros, así como la reflexión, son elementos que promueven el desarrollo de competencias. (Villardón, 2006). Por otra parte, el docente en este escenario, debe evaluar el proceso de aprendizaje y el desarrollo progresivo de los contenidos (Delgado et al., 2006).

El contexto en que se desarrolló la propuesta evaluativa, es el taller de diseño centrado en el usuario (DCU), que es una asignatura del tercer año de la carrera de Diseño Industrial, cuyo énfasis es diseñar un producto, basándose en requerimientos y necesidades de un usuario real en un contexto real (Zoe et al., 2012), considerando a éste desde inicio del proceso. En definitiva se pasa del diseñar "para la gente" a diseñar "con la gente". La temática solicitada en la propuesta de diseño, aborda la problemática social presente, a nivel país y regional, abordando la estrategia y enfoque de diseño inclusivo (Coleman et al., 2007), para considerar y trabajar con contextos en riesgo social de nuestro país, como es el caso Campamentos instalados en Coronel. Se aborda un trabajo colaborativo con el Centro de Innovación de "Un Techo para Chile" utilizando el diseño como articulador y generador de valor, y al estudiante de diseño como sujeto activo, constructor de su propia realidad (Good , 1996).

Los objetivos de la asignatura son: i) Aplicar metodologías de Diseño centradas en el usuario, considerando necesidades y requerimientos reales; ii) Aplicar un enfoque inclusivo con costos de producción bajos y especialización mínima de trabajo, para el diseño de un producto; iii) Manifestar el rol del Diseño con Responsabilidad Social; y iv) Aplicar herramientas de representación visual, que apoyen el desarrollo colaborativo, materialización y comunicación del proyecto.

La experiencia de conocer y trabajar con personas, y no con usuarios genéricos, permite al estudiante dimensionar la complejidad del ser humano y tener respuestas empáticas a sus necesidades. Así como, fortalecer su compromiso con la labor, a través de los lazos de confianza que van generando en este proceso, adscritos a un enfoque colaborativo, así el nuevo conocimiento se construye de manera conjunta y negociada (Martínez, 2003).

La Escuela de Diseño Industrial de la Universidad del Bío-Bío, como una forma de otorgar énfasis en el responsabilidad social, como competencia genérica institucional y que debe desarrollar el profesional Diseñador Industrial, ha generado una propuesta dual entre Diseño Centrado en el Usuario, la cual ubica al ser humano como centro inicio y fin del diseño (Krippendorff, 2006) y el Enfoque Proyectual (Munari, 2011) con una secuencia que orienta el proceso creativo para resolver un problema. Se considera como un enfoque más humanista y holístico. Por otro lado se asume la sistematización que proponen los métodos del diseño de ingeniería (Alcaide et al., 2001) para asistir y racionalizar la toma de decisiones. En consecuencia, utilizar la voz del usuario, desde una perspectiva participativa pasando desde el "diseño para" a un "diseño con" (Page et al., 2001). Siguiendo esta línea en la actualidad es cada vez es más común que se utilicen técnicas novedosas que se centren en investigar las necesidades del usuario para trasladarlas a los atributos del producto que se va a diseñar y sobre todo a cumplir las expectativas y deseos esperados (Gonzalez et al., 2009).

\section{METODOLOGIA}

La metodología y proceso proyectual desarrollado, se basa en las tres etapas esenciales (Antecedentes, Ideación y Construcción) planteadas por Bruce Archer (Archer en Cross 2008) que se puede apreciar en la figura 1. En la metodología, se plantea una estrategia de integración incluyendo etapas claves en la detección de problemas, priorización de necesidades, y generación de soluciones, se obtiene un modelo metodológico que se implementa dentro del taller de diseño, el que se ilustra en la figura 2. 


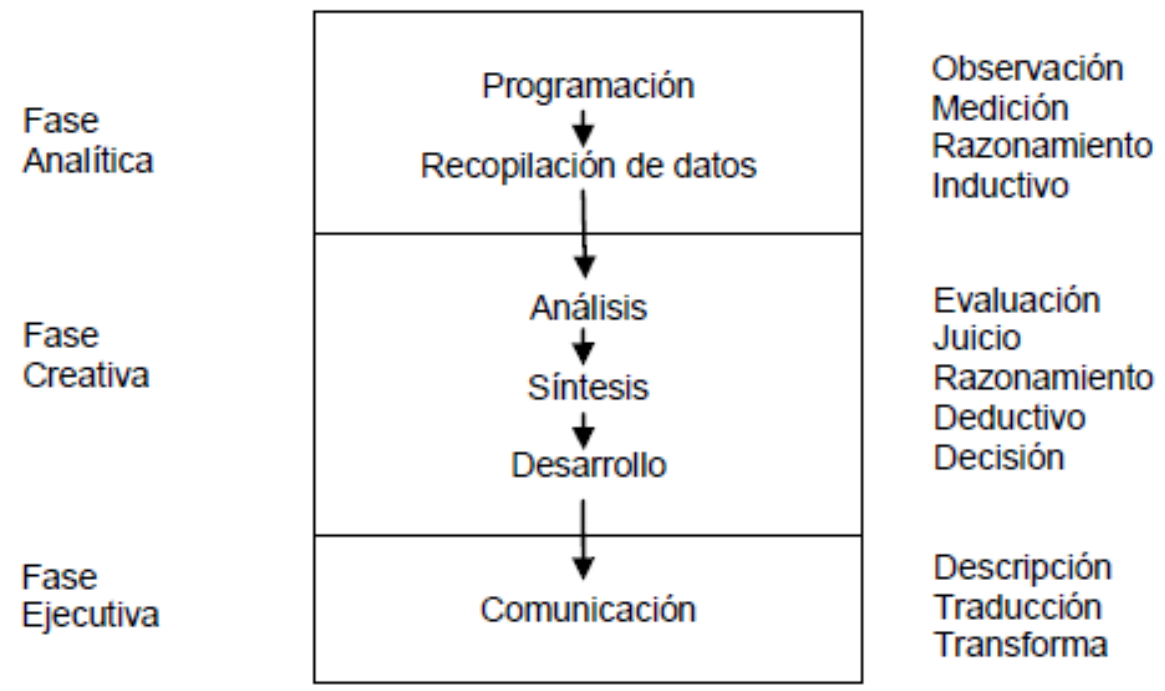

Fig.1: Esquema de Diseño Prescriptivo de Archer (1963) Fuente: Adaptado de Cross.

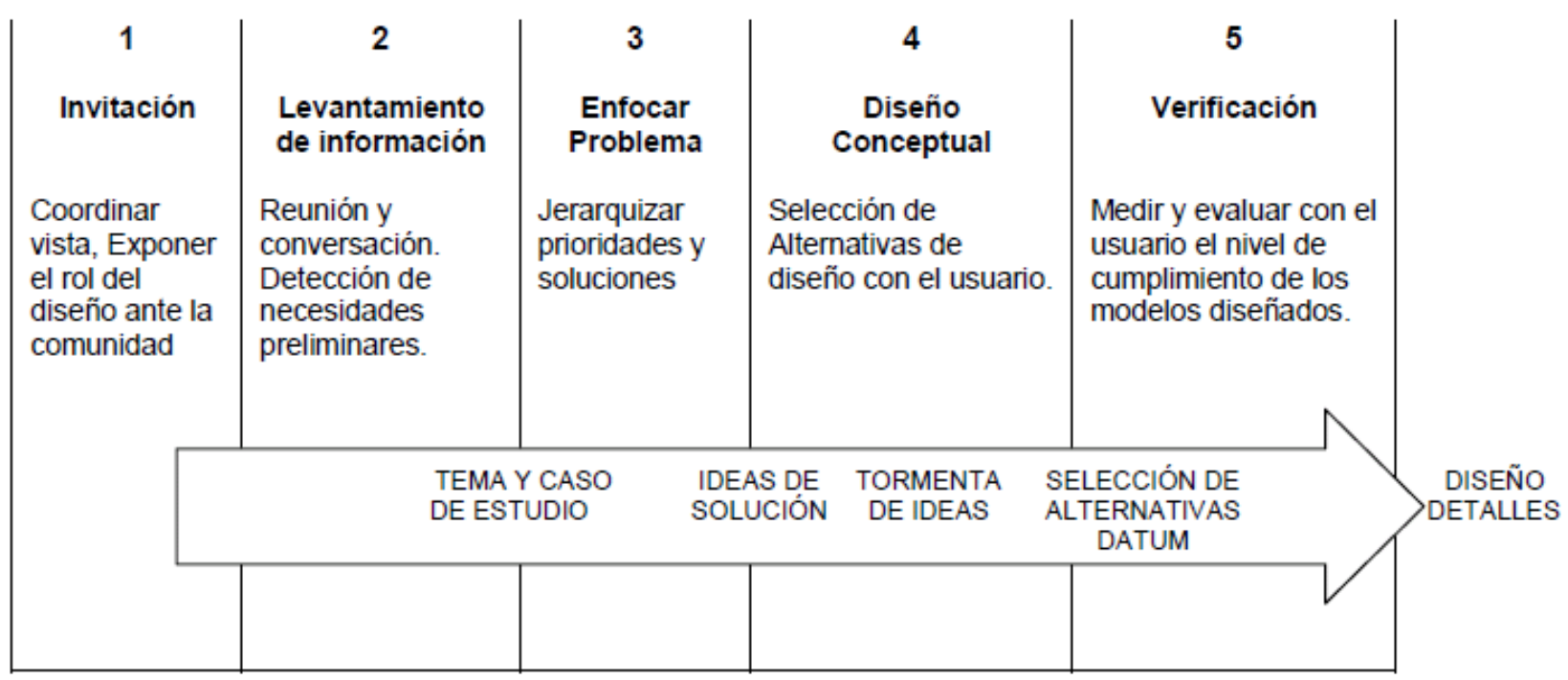

Fig. 2: Secuencia metodológica Diseño Centrado en el Usuario.

\section{FASES Y DESCRIPCIÒN DE LA SECUENCIA METODOLOGICA DE DCU}

Invitación

El objetivo es convocar a la comunidad, instancia en que se presentan las ideas del proyecto "Centrado en el Usuario", se expone el rol del Diseñador Industrial, éste se aproxima a la comunidad y a la realidad local, mediante el Centro de Innovación "Un Techo para Chile". Las preguntas orientadoras que acompañan esta etapa: ¿Cuál es el proyecto? (se explica a los usuarios) ¿cuál es el campo de acción en este proyecto específico?, si se trabajará en contexto de viviendas sociales, ¿qué tipo de problemas se presentan en este contexto? Se acotan los alcances y las soluciones, se establece el Rol del Diseñador Industrial Rol del Usuario. Esta etapa surge luego que se inicie el proceso de construcción de viviendas de emergencias en los contextos afectados. Un proceso de colaboración de diseño en acción como se puede apreciar en la figura 3. 

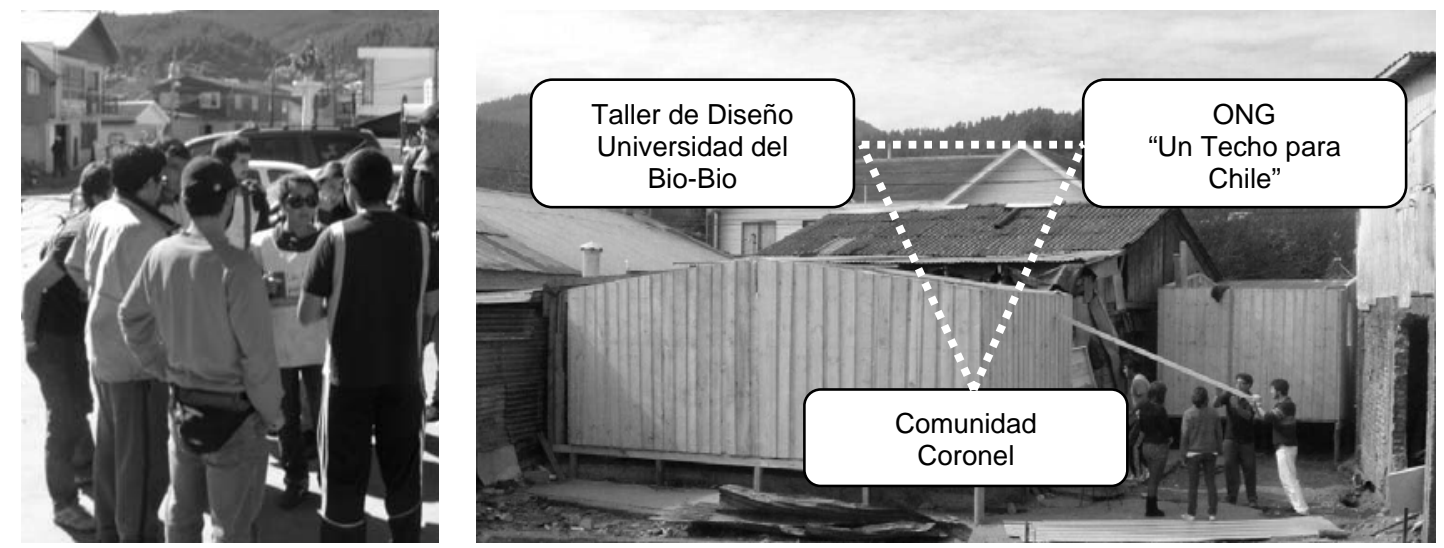

Fig. 3: Visita y construcción de viviendas de emergencias.

\section{Levantamiento de información}

Se recopila información acerca de los usuarios, perfil del usuario, y el reconocimiento de su contexto como se ilustra en la figura 4. A partir de técnicas de entrevista, se detectan necesidades genéricas de la comunidad en estudio, el resultado es un listado de problemas.

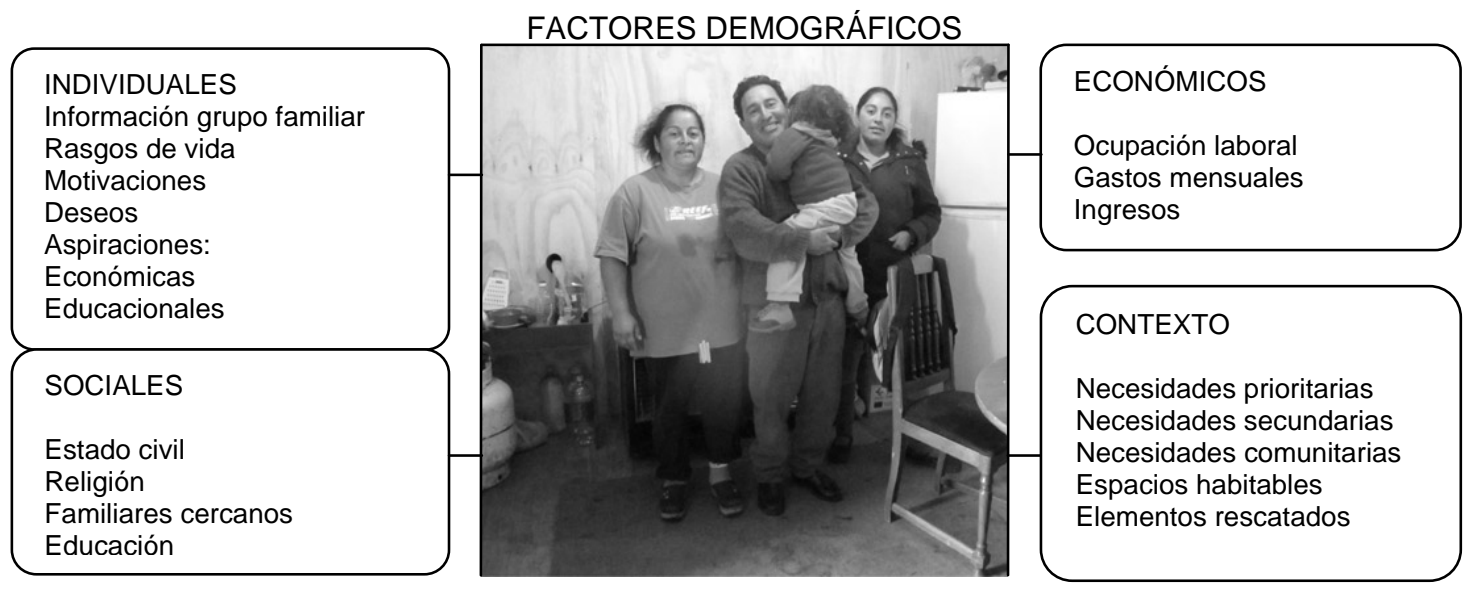

Fig. 4: Perfil y estudio del usuario.

\section{Enfocar /Definir el Problema}

Se determina el tema y caso de estudio, se acota el problema, se precisa, se jerarquiza prioridades y posibles soluciones, mediante metodología de clasificación de tarjetas (cardsorting), considerando listado de problemas, el usuario es quien, prioriza y evalúa la posibilidad de inversión, guiados por los estudiantes, como futuros Diseñadores Industriales este proceso finaliza con una mapa visual como se grafica en la figura 5 donde se ponderan las alternativas.

\section{Clasificación de tarjetas (card-sorting)}

1. Higiene personal

2. Separador ambiental para intimidad personal

3. Optimizar la aislación térmica

4. Secador de ropa para interiores

5. Mobiliario compactable para espacio reducido.

6. Minimizar la exposición de la basura

7. Autoabastecimiento: generar un huerto familiar

8. Optimizar preparación de alimentos

9. Guardado de prendas de vestir

10. Aislación térmica

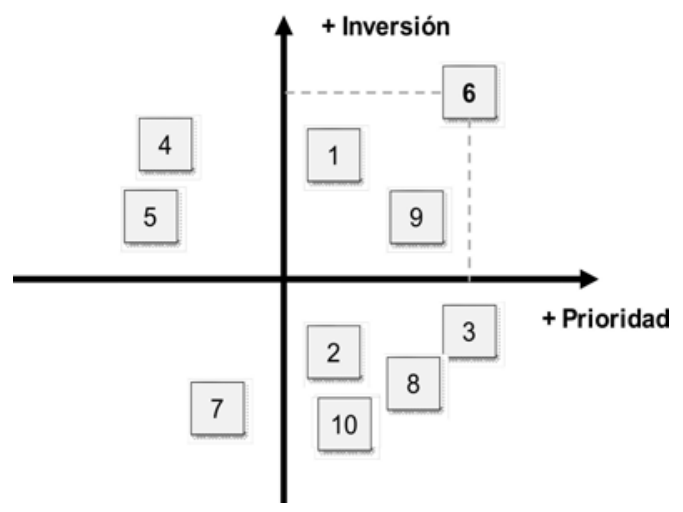

Fig. 5: Lámina de clasificación de tarjetas (card-sorting) para valorar problemáticas detectadas. 


\section{Diseño Conceptual}

Se desarrollan ideas o conceptos como posibles soluciones al problema. Se aborda paralelamente con la técnica de tormenta de ideas (brainstorming) donde los usuarios también pueden participar. Luego estas ideas se seleccionan. Se define una propuesta conceptual redactando en una frase las características y atributos que el producto deberá resolver utilizando respondiendo; el ¿qué es?, ¿Mediante qué? y el ¿para qué? En la figura 6 se ejemplifica el desarrollo formal y la propuesta formal de un contenedor de basura. Mediante el uso de bocetos se exploran diversas posibilidades formales y constructivas, definiendo aspectos de tamaño, usabilidad, función entre otros. Cotejando siempre en función de los objetivos y requisitos cual de las alternativas es la más pertinente hasta llegar a definir y refinar la propuesta formal seleccionada.
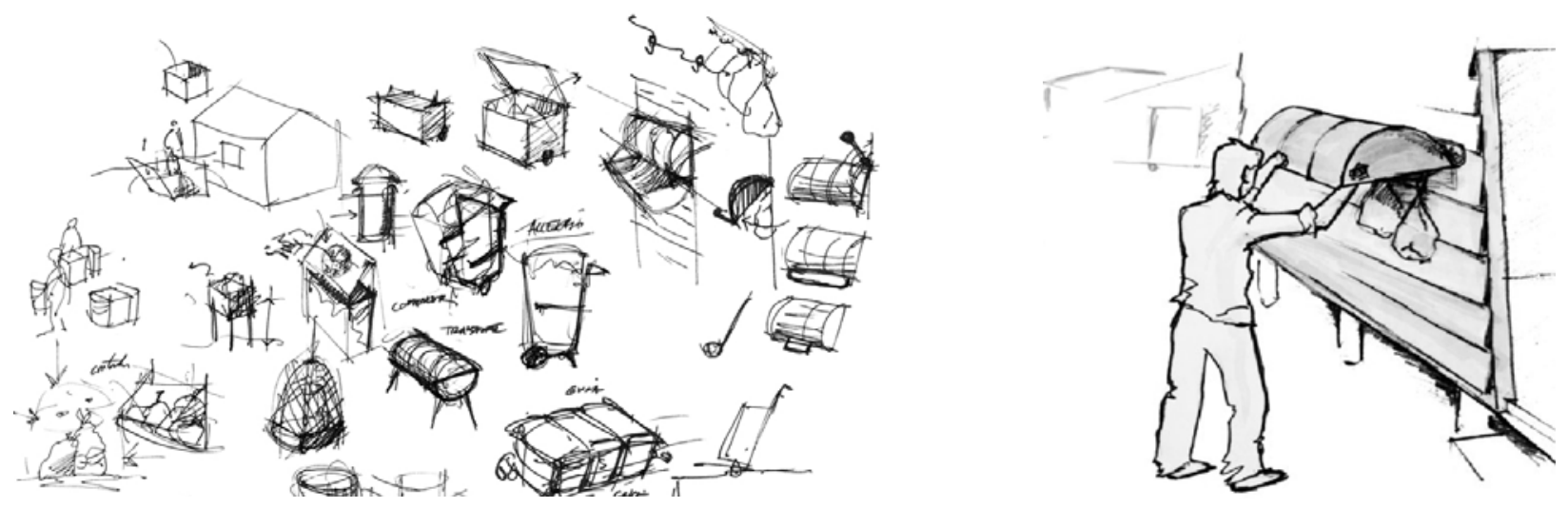

Fig. 6: Desarrollo Formal y Propuesta formal

\section{Verificación:}

Como parte del diseño conceptual, se seleccionan posibles alternativas, según criterios (asociados al producto, a las necesidades que debiera satisfacer, a la solución del problema, requerimientos y condicionantes que el objeto debería responder), se listan, se dibujan las alternativas, mediante método datum se verifica qué alternativa es la mejor opción para la solución del problema, mediante una maqueta y prototipo para verificar aspectos de usabilidad y función, que es ejemplificado en la figura 7.

\begin{tabular}{|l|l|}
\hline $\begin{array}{l}\text { A) Basurero } \\
\text { tipo baúl con } \\
\text { contenedor } \\
\text { removible. }\end{array}$ \\
\hline $\begin{array}{l}\text { B) Tanque } \\
\text { hermético } \\
\text { vertible. }\end{array}$ \\
\hline $\begin{array}{l}\text { C) Basurero } \\
\text { modular móvil. }\end{array}$
\end{tabular}

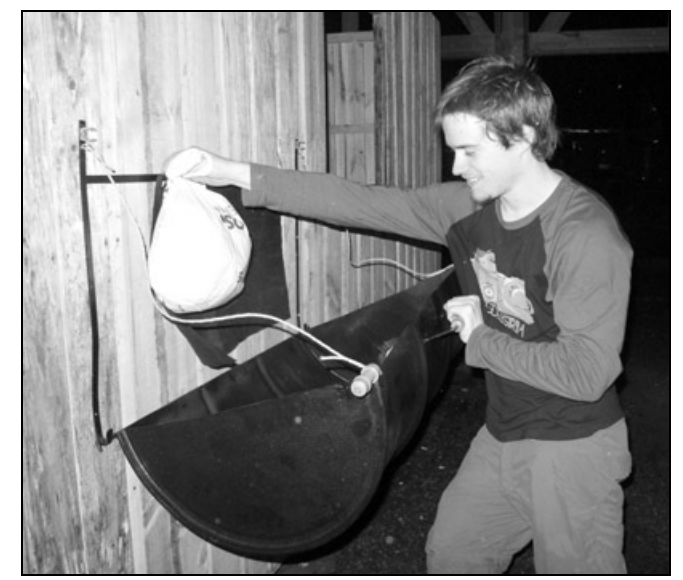

Fig. 7: Selección de alternativa y testeo de prototipo

\section{NUEVAS PROPUESTAS EVALUATIVAS}

\section{Evaluación de competencias}

En los actuales contextos de la formación universitaria, la evaluación debe ser considerada como una función relevante del currículum, coherente con la concepción curricular a la cual se adscribe, y considerar al estudiante como sujeto que participa del proceso de enseñanza aprendizaje, es decir como sujeto de 
significados y no sólo como sujeto aprendiente, por tanto la evaluación está inmersa en el proceso de enseñanza y aprendizaje, constituye una acción continua y consustancial, la cual debe ser analizada y reflexionada en relación con la innovación y con el uso estratégico en el aprendizaje. (Castro, 2011) Por tanto, el docente universitario es uno de los responsables de estimular en los estudiantes los conocimientos propios de la disciplina que enseña y las competencias que son necesarias para la adaptación en el mundo laboral, (Daura, 2011), tal como lo establece el Modelo Educativo de la Universidad del Bío-Bío, en cuanto al nuevo rol del docente y del estudiante en el enfoque centrado en el aprendizaje, incorporando el enfoque de evaluación como proceso y una evaluación auténtica, que se genera en un ambiente de aprendizaje que simule el desarrollo profesional futuro. Es así como el Taller de Diseño Centrado en el Estudiante, promueve el desarrollo de competencias que el profesional "Diseñador Industrial" debe desarrollar: Detectar problemas, solucionar problemas mediante propuestas de diseño innovadoras, e incluir a los sujetos en el proceso de acompañamiento de la propuesta para explorar sus verdaderas necesidades. Por ello los principales cambios en educación universitaria, es replantear los modelos evaluativos que se han ido aplicando hasta ahora. En este sentido, la exigencia de llevar a cabo una educación más integral, con mayor protagonismo de los estudiantes y basada en la adquisición de competencias de diferente naturaleza, en un contexto social cada vez más tecnificado, y que responde a nuevas necesidades, requiere de una revisión consciente y eficiente del diseño educativo de los programas universitarios. (Irigoyen et al., 2011). Así, las nuevas formas de evaluar, consideran los siguientes aspectos:

Exigir a los estudiantes tareas orientadas a que construyan sus respuestas más que a que las seleccionen. Recoger información que permita la observación directa de las ejecuciones de los estudiantes. Exigir que la naturaleza de las respuestas faciliten la iluminación de los procesos cognitivos y los aprendizajes implicados en las tareas a ejecutar (Mateo y Martínez, 2008). Es así como, en el modelo educativo orientado a competencias, utiliza la evaluación estratégicamente y de modo integrado con las actividades de aprendizaje y enseñanza y en él se debe producir una revaloración de la evaluación formativa-continua y una revisión de la evaluación final-certificativa (Fernández, 2011).

En consecuencia, no es posible hablar de innovar el proceso de aprendizaje-enseñanza sin una innovación paralela de la actividad evaluativa, puesto que, los estudiantes no modificarán su forma de aprender si sus aprendizajes van a seguir evaluándose según prácticas anteriores. Del mismo modo, los profesores no mejorarán su enseñanza, asumiendo una perspectiva profesional de su tarea, si no someten su enseñanza y sus prácticas educativas a procesos de evaluación que orienten la mejora y que, incluso, reconozcan la calidad docente. (Fernández, 2011). Los cambios evaluativos en este nuevo escenario, por tanto se pueden resumir en lo que establece (Zabalza, 2003): servir para ayudar a los alumnos a desarrollar sus capacidades, referirse a todos los objetivos formativos, esto es, ser integradora, estar inmersa en el desarrollo habitual del proceso enseñanza-aprendizaje, ser parte integrante del profeso formativo, ser coherente con el estilo de trabajo en el aula, ser inicial, de proceso y final, ser formativa, incluir demandas cognitivas variadas y progresivas, e incluir información previa y posterior.

\section{Indicadores del desempeño en el taller DCU}

La propuesta evaluativa del Taller DCU, tiene como punto de partida la competencia específica a desarrollar en el Diseñador Industrial, siendo evidencia de ella, el resultado de aprendizaje que es: "Diseñar una propuesta de proyecto o producto basado en necesidades y requerimientos de usuarios reales aplicando metodologías centradas en el usuario". Metodológicamente se estableció indicadores, en cada etapa del proyecto, los cuales son entregados a cada estudiante y consensuados en el taller de Diseño centrado en el usuario para su discusión y aceptación. El enfoque evaluativo al cual se adscribe es el de Evaluación Continua, generando procesos de evaluación formativa, según estados de avance de la propuesta, con énfasis en niveles de desempeño.

\section{RESULTADO DE APRENDIZAJE}

Para estructurar los resultados de aprendizajes se genera una Pregunta Orientadora: ¿Qué tiene que saber hacer los estudiantes de Diseño en el Taller de Diseño Centrado en el Usuario? Finir el macro propósito del aprendizaje. En este caso sería: Diseña un producto basado en necesidades y requerimientos de usuarios reales aplicando metodologías centradas en el usuario. Asi la rúbrica de evaluación de la tabla 1 se basa en los aspectos (indicadores) que el alumno deberá resolver como parte del proceso de diseño. 
Tabla1: Rubrica de evaluación.

\begin{tabular}{|c|c|c|c|}
\hline Indicadores & \multicolumn{3}{|c|}{ Descriptores } \\
\hline \multirow{3}{*}{$\begin{array}{l}\text { Definición de } \\
\text { Problema } \\
15 \%\end{array}$} & Experto (100-90 ) & Intermedio (89- 65) & Novato (64- 1) \\
\hline & $\begin{array}{l}\text { Establece claramente el objeto y } \\
\text { sujetos del problema, contextualiza } \\
\text { con precisión la realidad local y } \\
\text { análoga de país, mediante el estado } \\
\text { del arte, que permita un impacto a la } \\
\text { comunidad. }\end{array}$ & $\begin{array}{l}\text { Establece con ciertas } \\
\text { impresiones el objeto y } \\
\text { sujetos del problema, sólo } \\
\text { contextualiza la realidad } \\
\text { local. }\end{array}$ & $\begin{array}{l}\text { Establece solo los sujetos } \\
\text { del problema y con } \\
\text { ambigüedades el objeto, } \\
\text { contextualiza algunos } \\
\text { elementos del entorno. }\end{array}$ \\
\hline & $\begin{array}{l}\text { Establece claramente registros de } \\
\text { aplicación de técnicas del diseño } \\
\text { clasificación de tarjetas a partir de la } \\
\text { retroalimentación del usuario. }\end{array}$ & $\begin{array}{l}\text { Establece registros de aplica } \\
\text { algunas técnicas del diseño: } \\
\text { clasificación de tarjetas a } \\
\text { partir de la retroalimentación } \\
\text { del usuario. }\end{array}$ & $\begin{array}{l}\text { Establece con omisiones } \\
\text { los registros de aplicación } \\
\text { de técnicas del diseño } \\
\text { clasificación de tarjetas, no } \\
\text { se presenta una } \\
\text { retroalimentación entre el } \\
\text { diseñador y el usuario. }\end{array}$ \\
\hline $\begin{array}{l}\text { Objetivos } \\
15 \%\end{array}$ & $\begin{array}{l}\text { Se presenta el objetivo general y } \\
\text { específico del proyecto vinculándolos } \\
\text { con el problema, usabilidad, } \\
\text { funcionalidad. }\end{array}$ & $\begin{array}{l}\text { Se presenta el objetivo } \\
\text { general y específicos del } \\
\text { proyecto, se vincula con } \\
\text { algunas impresiones la } \\
\text { usabilidad, funcionalidad. }\end{array}$ & $\begin{array}{l}\text { Se presenta el objetivo } \\
\text { general y específicos del } \\
\text { proyecto, no se vincula con } \\
\text { la usabilidad y } \\
\text { funcionalidad. }\end{array}$ \\
\hline $\begin{array}{l}\text { Diseño } \\
\text { Conceptual } \\
\text { Lluvia de ideas } \\
10 \%\end{array}$ & $\begin{array}{l}\text { Se establece claramente ideas, } \\
\text { conceptos, como soluciones al } \\
\text { problema mediante lluvia de ideas }\end{array}$ & $\begin{array}{l}\text { Se establece con ciertas } \\
\text { impresiones las ideas, } \\
\text { conceptos, como soluciones } \\
\text { al problema mediante lluvia } \\
\text { de ideas. }\end{array}$ & $\begin{array}{l}\text { Las ideas, conceptos, no } \\
\text { constituyen verdaderas } \\
\text { soluciones al problema, } \\
\text { utiliza con imprecisiones la } \\
\text { técnica de lluvia de ideas. }\end{array}$ \\
\hline $\begin{array}{l}\text { Propuesta } \\
\text { Conceptual } \\
17 \%\end{array}$ & $\begin{array}{l}\text { Se establecen claramente los } \\
\text { atributos, definiendo: el qué, para qué } \\
\text { y el mediante qué del objeto. }\end{array}$ & $\begin{array}{l}\text { Se establecen algunos } \\
\text { elementos como atributos } \\
\text { que definen: el qué, para } \\
\text { qué y mediante qué del } \\
\text { objeto. }\end{array}$ & $\begin{array}{l}\text { Se establece con } \\
\text { ambigüedades el qué, para } \\
\text { qué y mediante qué del } \\
\text { objeto. }\end{array}$ \\
\hline $\begin{array}{l}\text { Desarrollo } \\
\text { Formal } \\
17 \%\end{array}$ & $\begin{array}{l}\text { Presenta diversas alternativas para la } \\
\text { solución del problema considerando } \\
\text { amplitud de configuraciones } \\
\text { morfológicas }\end{array}$ & $\begin{array}{l}\text { Presenta algunas } \\
\text { alternativas para la solución } \\
\text { del problema considerando } \\
\text { exploración parcial de } \\
\text { propuestas }\end{array}$ & $\begin{array}{l}\text { Presenta con } \\
\text { ambigüedades } \\
\text { alternativas para la } \\
\text { solución del problema }\end{array}$ \\
\hline $\begin{array}{l}\text { Propuesta } \\
\text { Formal } \\
17,5 \%\end{array}$ & $\begin{array}{l}\text { Desarrolla las características formales } \\
\text { del producto, considerando materiales, } \\
\text { formas, tamaños, atributos, partes, } \\
\text { piezas, usabilidad y función, } \\
\text { acercándose a la solución del } \\
\text { problema. }\end{array}$ & $\begin{array}{l}\text { Desarrolla las características } \\
\text { formales del producto, } \\
\text { considerando algunos } \\
\text { elementos como: materiales, } \\
\text { formas, tamaños, atributos, } \\
\text { partes y piezas, usabilidad y } \\
\text { función, acercándose a la } \\
\text { solución del problema }\end{array}$ & $\begin{array}{l}\text { Desarrolla las } \\
\text { características formales del } \\
\text { producto, con } \\
\text { imprecisiones: materiales, } \\
\text { formas, tamaños, partes y } \\
\text { piezas, atributos, } \\
\text { usabilidad y función, } \\
\text { acercándose a la solución } \\
\text { del problema. }\end{array}$ \\
\hline $\begin{array}{l}\text { Verificación } \\
17,5 \%\end{array}$ & $\begin{array}{l}\text { Los atributos planteados a nivel } \\
\text { conceptual del producto se } \\
\text { materializan solucionando aspectos } \\
\text { formales, funcionales y de usabilidad } \\
\text { de manera integrada. }\end{array}$ & $\begin{array}{l}\text {.Algunos atributos y } \\
\text { características están } \\
\text { consideradas de manera } \\
\text { desagregada. Presentando } \\
\text { ciertas incoherencias entre } \\
\text { la forma, función y } \\
\text { usabilidad. }\end{array}$ & $\begin{array}{l}\text { El producto responde de } \\
\text { manera parcial e } \\
\text { incompleta frente a los } \\
\text { atributos planteados. No } \\
\text { hay coherencia entre los } \\
\text { conceptos y la } \\
\text { materialización del objeto. }\end{array}$ \\
\hline
\end{tabular}


Taller

[DCU]

\section{Tema:}

Almacenamiento de la ropa de uso

frecuente.

\section{Contexto:}

Campamento "La Aldea", Penco chico.

Necesidad:

Separar la ropa interior por razones

socio-culturales referidos a la sexualidad de

los ocupantes de una pieza.

\section{Caso:}

Familia completa que duerme sólo en

una pieza de mediagua.

\section{Oportunidad:}

Intimidad dada por el ocultamientoa

causa del pudor femenino.

\section{Problemática:}

Mezcla de la ropa al haber un único

punto de almacenaje.

\section{Propuesta Conceptual:}

"Estuche moldeable de posición adaptable para el ocultamiento de la ropa interior femenina"

\section{Observaciones:}

“Para proveer seguridad en la propiedad privada, el elemento femenino recurre a ocultar la ropa su ropa interior en una bolsa como unico método para crear intimidad en al pieza".

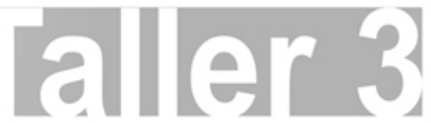

Saturación a causa de

Saturación a causa de la convergencia externa en el lugar de almacenemiento, genera confusión de limites de propiedad en la vestimenta.
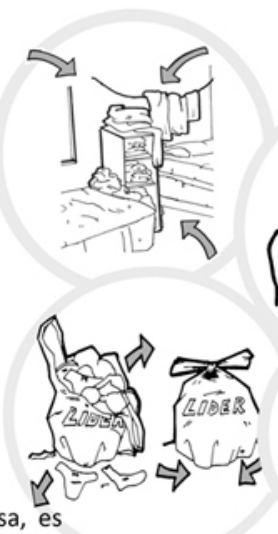

guardado en una bolsa, es porque al ser semejante a una membrana, esta puede expandirse o contraerse sin perder la calidad del ocultamiento gracias a su elasticidad.
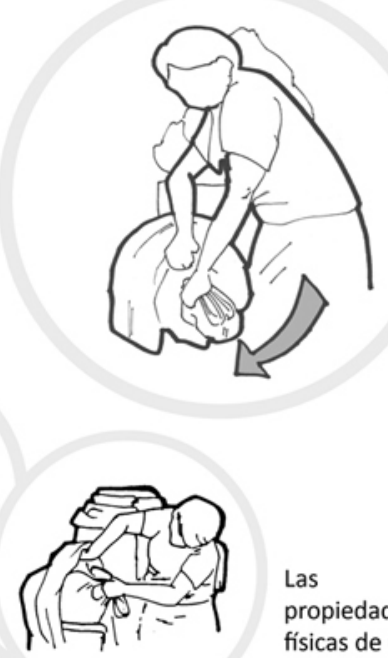

Las

propiedaddes físicas de una bolsa plástica, da con un mejor ocultamiento, por ser un límite moldeable, porque tiene la capacidad de adaptarse a cuelquier espacio.

\section{Propuesta Formal:}
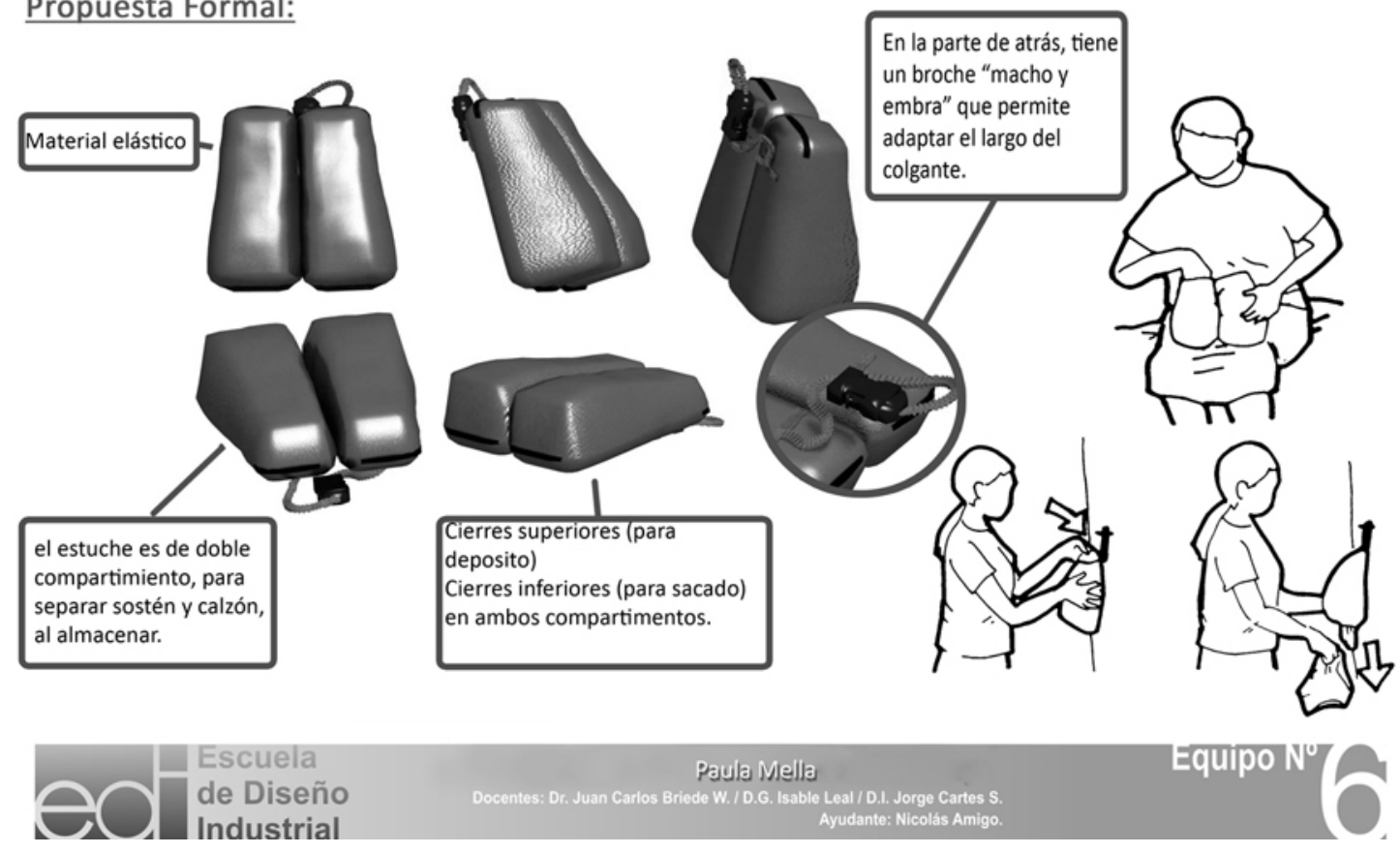

Fig.8: Ejemplo lámina presentación proyecto taller de diseño IIIํaño 2011. Elaboración: Paula Mella. 


\section{CONCLUSIONES}

La propuesta evaluativa, se determinó mediante el análisis y descripción de cada fase del diseño de un producto, considerando el enfoque disciplinario, Diseño Centrado en el Usuario y la metodología Proyectual, para establecer indicadores evaluativos vinculados con los resultados de aprendizaje planteados por el programa del taller y su vinculación con las competencias específicas y genéricas de la carrera de Diseño Industrial, enfatizando en la competencia genérica de Responsabilidad Social puesto que el estudiante de Diseño Industrial, se involucra con un contexto vulnerable de la región del Bío-Bío.

El DCU, permite vincular al estudiante en forma colaborativa con sus pares, con el contexto al cual él se desempeñará en su vida profesional futura, por lo cual, también se incorpora como descriptor en la rúbrica, incluyendo la visión humana del Diseño.

Establecer un vínculo entre estudiantes-comunidad, permite que el diseño de un producto se elabore con necesidades y requerimientos reales, así también contribuye al desarrollo de las competencias disciplinarias y transversales, del Diseñador Industrial puesto que se desenvuelven en contextos reales como futuros profesionales.

Contar con una rúbrica de evaluación, permite mejorar los procesos de aprendizaje y evaluativos, puesto que se describe con objetividad qué es lo que el estudiante debe desarrollar para alcanzar el resultado de aprendizaje que el taller establece y así la formación de competencias que la carrera declara.

\section{AGRADECIMIENTOS}

Esta publicación es parte del proyecto de Investigación DIUBB 110404 2/R y fue desarrollada gracias al apoyo de la Dirección de Investigación del la Universidad del Bío-Bío.

\section{REFERENCIAS}

Alcaide, J., Diego, J., M. Artacho, Diseño de Productos. Métodos y técnicas, $1^{\text {a }}$ edición,19-378. Editorial Universidad Politécnica de Valencia, Valencia,España (2001).

Cano, M., La Evaluación por Competencias en la Educación Superior, Revista de Currículum y Formación del Profesorado, ISSN: 1138-414X, (en línea), 12 (3), 2008. http://www.ugr.es/ recfpro/rev123COL1.pdfAcceso: 11 abril (2012).

Castro, F., ¿Por qué las universidades deberían cambiar sus prácticas evaluativas? Hacia el reconocimiento de la voz del sujeto de la evaluación. Praxis Educativa: 16 (14), 94-99 (2011).

Coleman, R.,Clarks J.,Dong H., J. Cassim. Design for Inclusivity. A Practical Guide to Accesible Innovative an User-Centred Design, $1^{\text {a }}$ edición, 1-246. Gowe Publishing Limited,Hampshire.England (2007).

Cross N., Engineering Design Methods: Strategies for Product Design, $4^{\mathrm{a}}$ edition, 1-159. John Wiley and Sons, Chichester, UK (2008).

Daura, F.. Las estrategias docentes al servicio del desarrollo del aprendizaje autorregulado. Estudios Pedagógicos : 37 (2), 77-88 (2011).

Delgado, M. y R. Oliver, La Evaluación continua en un nuevo escenario docente. Revista Universidad y Sociedad del Conocimiento, ISSN: 1698-580X (en línea) 3 (1), 2006, http://www.uoc.edu/rusc/3/1/dt/esp/delgado_oliver.pdf- Acceso: 11 abril (2012).

De Vicenzi, A., P. De Angelis, La evaluación de los aprendizajes de los alumnos. Orientaciones para el diseño de instrumentos de evaluación, Educación y Desarrollo : 8, 17-22 (2008).

Fernández, A., La innovación de la docencia como estrategia de cambio ante los desafíos del EEES. Universidad Politécnica de Valencia. Experiencias Docentes y Tics. ICE. Universidad de Oviedo. $1^{\mathrm{a}}$ edición,Ediciones Octaedro, 7-341. Barcelona, España (2011).

Flavell, J. H., Metacognición and cognitive monitoring: A new area of cogntive-developmental inquiry, American Psychologist: 34(10) 906-911 (1979). 
Gonzalez, M., J.R., Lama, J.R. Pérez,Ingeniería Kansei para un diseño de producto centrado en el usuario, Rev.Técnica Industrial: 280 (1), 68-74(2009).

Good, T. Psicología Educativa Contemporánea, 1ª edición, 7-575. Mc Graw Hill. México, D.F. (1996).

Irigoyen, J. J., M. Y., Jiménez, y K. F. Acuña, Competencias y educación superior, Revista mexicana de investigación educativa: 16(48), 243-266 (2011).

Krippendorf K. The semantic turn: a new foundation for design, $1^{a}$ edition, 1-349. Taylor\& Francis Group, Boca Raton, USA (2006).

Lim, Z., C. Anderson, y S. McGrath, Professional Skills Development in a Resource-Poor Setting: The Case of Pharmacy in Malawi, International Journal of Education Development: 32 (5), 654-664 (2012).

Martínez , F. Redes de Comunicación en la Enseñanza: las nuevas perspectivas del trabajo corporativo, $1^{\mathrm{a}}$ edición, 9-310. Paidós,Barcelona, España (2003).

Mateo, J. y F. Martínez, Medición y evaluación educativa. 1ª edición, 9-309. La Muralla, Madrid, España (2008).

Munari B. ¿Cómo nacen los objetos? Apuntes para una metodología proyectual, 14 a edición,5- 388. Gustavo Gili, Barcelona, España (2011).

Munari, B. Fantasia invenzione, creativitá e immaginazione nelle comunicación visive, $10^{\mathrm{a}}$ edición, 5-219. Editori Laterza ,Roma, Italia (2006).

Ospina, A., Currículo por competencias en la Universidad de La Sabana, Aquichan: 6 (1) 117-124 (2006).

Page, A., R. Pocar, M.J. Such, J. Solaz, V., Blasco,Nuevas técnicas para el desarrollo de productos innovadores orientados al usuario, $1^{\mathrm{a}}$ edición, 4-124. Editorial Instituto de Biomecánica de Valencia y ADCV ,Valencia, España (2001).

Perrenoud. P., Diez nuevas competencias para enseñar, $3^{\text {a }}$ edición, 7-155. Editorial Graó, Barcelona,España (2005)

Perrenoud, P. ,La evaluación de los alumnos. De la producción de la excelencia a la regulación de los aprendizajes. Entre dos lógicas, $1^{\mathrm{a}}$ edición, 7-89. Ediciones Colihue,Buenos Aires,Argentina (2008).

Peters, M., Does Constructivist Epistemology Have a Place in Nurse Education?, Journal of Nursing Education: 39 (4) 166-170 (2000).

Regalado, A., Peralta, E. y Baez, J., Aprendizaje Basado en Competencias Aplicado a una Asignatura de Transferencia de Calor. Form. Univ, ISSN: 0718-5006 (en línea) ,4 (1), 13-18, 2011. http://www.scielo.cl/scielo.php?script=sci_arttext\&pid=S0718-50062011000100003\&lng=es\&nrm=iso>, doi: 10.4067/S0718-50062011000100003. Acceso: Noviembre 2012.

Sanoff, H. Participatory Design: Theory and Techniques, $1^{a}$ edición, 5-210. Bookmasters, England, London (1990).

Sateke, R.E. Evaluación comprensiva y evaluación basada en estándares, $1^{\circ}$ Edición, 9-165. Graó, Barcelona, España, (2006).

Villardón, L. Evaluación del Aprendizaje para promover el desarrollo de competencias. Universidad de Deusto. Educación siglo XXI : 24 ,57-76(2006).

Yániz, C y L. Villardón , Planificar desde competencias para promover el aprendizaje, $1^{\text {a }}$ edición, 11-125. Editorial Mensajero, Bilbao, España (2006).

Zalba, E. M., Competencias para el ingreso y permanencia en la Universidad: una propuesta para la articulación curricular entre el nivel superior y el nivel medio de enseñanza. Actas del Seminario Currículo Universitario basado en Competencias, CINDA - Universidad del Norte, 2-351. Barranquilla, Colombia, 25 y 26 de Julio (2005). 